\title{
Endoscopic Sinus Surgery for Treatment of Kartagener Syndrome: A Case Report
}

\author{
Xinghua Tang, Jian Zou, Shixi Liu \\ Department of Otorhinolaryngology-Head and Neck Surgery, West China Hospital of Sichuan University, Chengdu, China
}

\begin{abstract}
Background: Kartagener syndrome (KS) is a rare congenital disease characterised by a clinical triad of symptoms: situs inversus, chronic rhinosinusitis, and bronchiectasis. Although congenital ciliary defect is recognised as the main cause of this syndrome, it remains difficult to treat the associated airway infection.

Case Report: A 17-year-old female patient presented with repeated refractory airway infection. She also had bronchiectasis and situs inversus. Electron microscopic evaluation of her nasal mucosa revealed ciliary defect and confirmed the diagnosis of KS. She underwent functional endoscopic sinus surgery (FESS) followed by long-term postoperative debridement of the sinonasal cavity. This treatment reduced chronic rhinosinusitis and protected against subsequent airway infection in a 7-year follow-up.
\end{abstract}

Conclusion: FESS is effective for relieving both chronic rhinosinusitis and lung infection of KS in the long term.

Key Words: Kartagener syndrome, primary ciliary dyskinesia, sinusitis, surgery, endoscopy

Received: 14.11 .2012

Accepted: 05.02.2013

\section{Introduction}

Kartagener syndrome (KS) is a rare congenital disease characterised by a triad of situs inversus, chronic rhinosinusitis (CRS), and bronchiectasis resulting from abnormalities in ciliary structure and/or function (1). Chronic and/or repeated airway infection presents from childhood and is difficult to treat; this condition then leads to CRS and bronchiectasis, aplasia or hypoplasia of the sinuses, and ultimately to impaired function of the lung and/or heart (2). There is no evidence-based strategy to manage airway infection in patients with KS. Pharmacological treatment often fails, but surgery may prove valuable in reducing KS symptoms. Herein, we report the case of a KS patient who underwent endoscopic sinus surgery and long-term postoperative debridement of the sinonasal cavity to control airway infection. The use of endoscopic surgery for the treatment of KS is described.

\section{Case Report}

A 17-year-old female patient presented with a history of nasal obstruction, mucopurulent rhinorrhoea, coughing, and expectoration for the previous 15 years. Her family history was not significant. Her parents reported that she was susceptible to rhinitis and prone to pneumonia when she was a baby. Antibiotic use was not as effective as expected for these conditions. Frequent visits to the paediatrician (every 2 to 3 months) and hospitalisation (every 3 to 4 months) were necessary. As she grew up, the need for hospitalisation decreased.
However, coughing with expectoration of purulent sputum started increasing gradually. She received cephalosporins orally and intravenously, and some other medications such as ambroxol, myrtol standardised, and ephedrine hydrochloride nasal drops for fever associated with coughing, nasal obstruction, and purulent sputum/rhinorrhoea. Acute airway infection exacerbated four to six times a year. When the symptoms of airway infection were alleviated, long-term intranasal steroids and oral antibiotics such as roxithromycin or clarithromycin were administered. Other drugs such as expectorants and Chinese herbs in combined formulas, including Angelicae Dahuricae Radix, Asiasari Radix, Ligusticum wallichii, Magnoliae Flos and others, were used intermittently. However, coughing with expectoration of white-yellowish sputum, intermittent nasal obstruction, and mucopurulent rhinorrhoea persisted. Nasal endoscopic examination revealed excess pus in both nasal cavities with oedema and chronic congestion of the inferior and middle turbinates. No polyps were observed. Computed tomography (CT) of the sinuses (Figure 1) revealed diffuse mucosal thickening of the right and left maxillary and ethmoid sinuses, and dysplasia of the frontal and sphenoid sinuses. An electrocardiogram revealed nodal rhythm, right electric axis deviation, and extreme clockwise transposition. Chest radiography showed that the cardiac silhouette and stomach air were located on the right side. These findings were suggestive of KS. CT of the thorax (Figure 2) confirmed bronchiectasis and facilitated the clinical diagnosis of KS. Because pharmacological treatment was ineffective, the patient was indicated for functional endoscopic sinus surgery (FESS) under local anaesthesia. The ethmoid cells were eradicated 


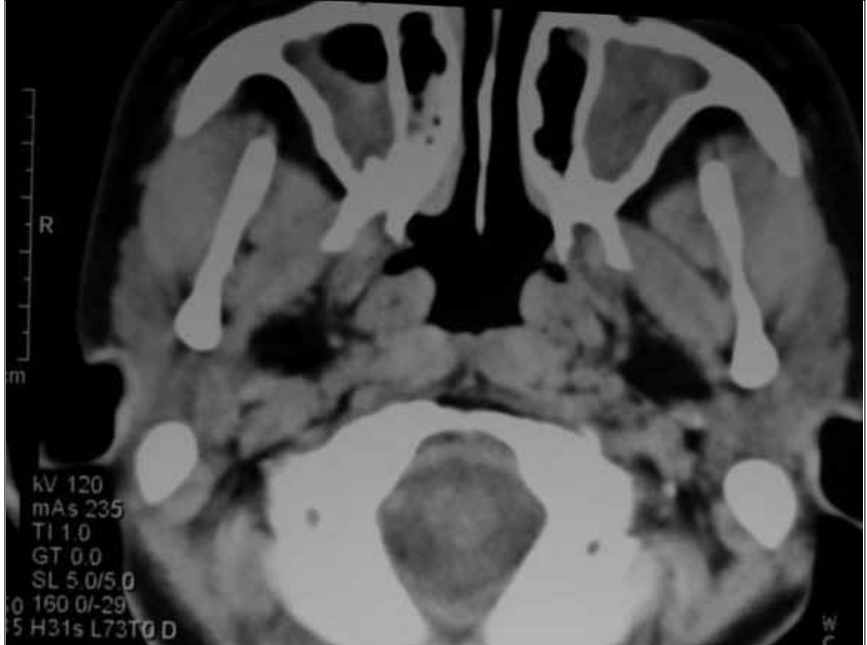

Figure 1. CT scan showing mucosal thickening in both maxillary sinuses

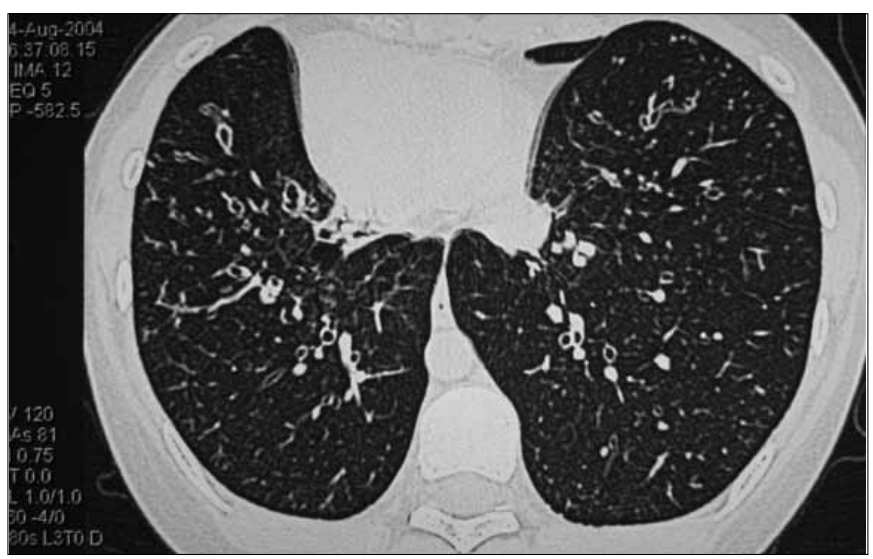

Figure 2. CT scan of thorax showing bilateral bronchiectasis

completely, and the natural ostia of the right and left frontal, maxillary, and sphenoid sinuses were enlarged. A mucosal sample was obtained from the ostium of the right maxillary sinus for electron microscopic examination. Scanning electron microscopic examination (Figure 3 ) showed that nasal mucosal cilia appeared normal and well organised. Transmission electron microscopic examination (Figure 4) showed that the inner and outer dynein arms of the cilia were missing; however, the ' $9+2$ ' structure of nasal cilia was normal.

The patient was discharged home 3 days after surgery. She used intranasal corticosteroids for approximately 3 months. The patient was followed up for routine postoperative endoscopic debridement of the sinonasal cavity; this procedure was initially performed weekly and then the frequency was gradually tapered to semi-monthly, monthly, trimonthly, semiannually, and then annually. Symptoms such as nasal obstruction and mucopurulent discharge were resolved. Coughing and expectoration were alleviated to a great extent but were occasionally aggravated by rhinitis. Two years after surgery, the ostia of both nasal sinuses were opened wide, but some mucus could still be seen in the antra. At the time of this report, 7 years had passed since surgery. Lung infection episodes were reduced to one or two times a year, with no need

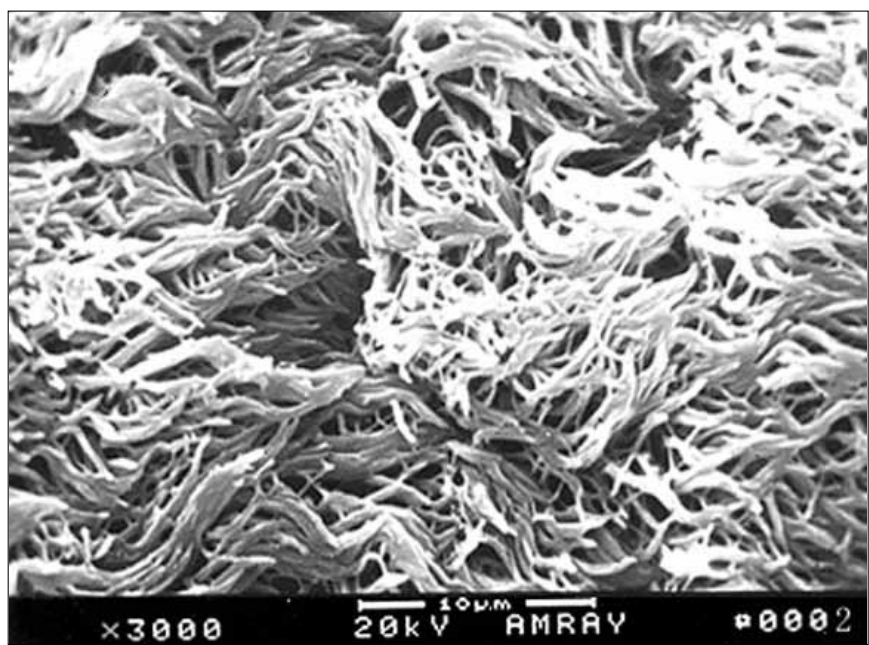

Figure 3. Scanning electron microscopic examination showing that nasal mucosal cilia appear normal and well organised

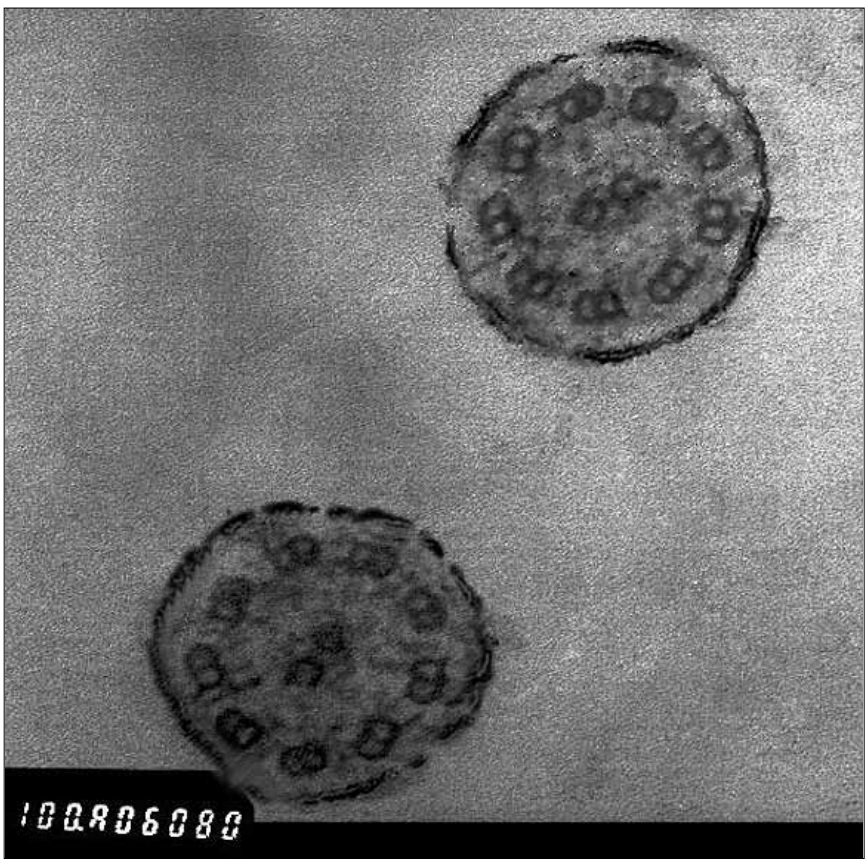

Figure 4. Transmission electron microscopic examination showing normal ' $9+2$ ' structure without the inner and outer dynein arms of the cilia

for hospitalisation. The severity and course of airway infection were reduced as well.

\section{Discussion}

KS is a clinical subgroup of primary ciliary dyskinesia caused by well-defined structural and/or functional changes in the motile cilia. During embryogenesis, ciliary dysfunction leads to random lateralisation of organs, e.g., situs inversus. After birth, it leads to reduced mucociliary clearance, and retained secretions increase the risk of infection in the airways and middle ear. In the present case, nasal purulent secretions, mucosal congestion, and swelling resulting from chronic infec- 
tion were evident. In men, infertility is often seen because the structure of the sperm tail is similar to that of a motile cilium (3). However, because the early symptoms of KS are nonspecific, the diagnosis of this condition is challenging.

$\mathrm{KS}$ is often misdiagnosed or diagnosed late, at an advanced stage, because of the following reasons: (a) KS is a rare congenital disease, and many clinicians are unaware of it; and (b) patients with KS are born with situs inversus but without CRS or bronchiectasis; the incidence of CRS or bronchiectasis increases with age as a result of chronic and/or recurrent airway infection (2). Therefore, the true incidence of KS may be higher than reported. Delayed diagnosis contributes to the progression of KS with complications such as aplastic or hypoplastic nasal sinuses, bronchiectasis, pulmonary fibrosis, and ultimately impaired lung and/or heart function; these conditions negatively impact the outcome. In the present case, KS was not diagnosed until the patient was 17 years of age, although she had frequent visits to physicians because of repeated airway infection. Advanced complications such as dysplasia of the frontal and sphenoid sinuses, CRS, and bronchiectasis occurred in the present case. Pifferi et al. (4) observed aplasia/hypoplasia of the frontal and/ or sphenoidal sinuses in $30(73 \%)$ of 41 patients with primary ciliary dyskinesia. Aplasia of the frontal sinus is present unilaterally only in $15 \%$ and bilaterally in $5 \%$ of normal adults, and aplasia of the sphenoid sinus is rare (5). The frontal and sphenoidal sinuses, which grow postnatally, are commonly involved in aplasia/hypoplasia, suggesting that aplasia/hypoplasia of sinuses is associated with chronic infection and inflammation in patients with KS. Chronic infection and inflammation may induce premature ossification of the cartilage surrounding the paranasal recesses and induce failure of the invasion of nasal mucosal epithelium into the surrounding nasal capsule cartilages $(6,7)$. Recurrent lung infections induce inflammatory and destructive changes in the bronchial wall. Normal structural components, such as cartilage, muscle, and elastic tissue, are destroyed and replaced by fibrous tissue, and eventually bronchiectasis occurs. The latter phenomenon was observed in the present case. Early diagnosis and effective intervention may prevent or at least delay some of the above-mentioned complications. Therefore, it is necessary to correctly diagnose KS at an early stage.

Electron microscopic examination of mucosal cilia helps in the diagnosis of KS. The airway mucosa is covered by many ciliated cells, each projecting approximately 200 cilia into the airway (1). More than 200 proteins and polypeptides are involved in ciliary formation and structure (1). Most of these are components of specific axonemal structures that can be visualised clearly by electron microscopic examination. The ultrastructure of a normal cilium consists of an axoneme, the core of respiratory cilium, and a pair of central singlet microtubules surrounded by nine pairs of doublets (the ' $9+2$ ' pattern) (1). Other structures such as the bridge connecting central singlets, central sheath, radial spoke, outer and inner dynein arm, nexin link, and ciliary membrane are all very important to maintain the normal structure and function of the cilium. Any abnormality-or a combination of abnormalities-of these structures may impair ciliary function. In the current case, the absence of outer and inner dynein arms was observed; this confirmed the diagnosis of KS. However, elec- tron microscopic examination alone is insufficient to detect KS because some patients with a clinical picture compatible with KS do not have ultrastructural ciliary defects on electron microscopic examination but do have abnormal mucociliary motility (8). Further studies are required to investigate this phenomenon in $\mathrm{KS}$.

It is difficult to control airway infection in patients with KS. Evidence-based treatment strategies are unavailable because of limited data (9). The current therapeutic strategy for KS includes medication, surgery, and some adjuvant therapies. Medication is the major choice and should be carried out early and effectively. It focuses on modifying mucus viscosity and controlling inflammation and infection in the airway (10), and includes mucoactive agents, steroids, and antibiotics. Adjuvant therapy includes nasal irrigation, influenza and pneumococcal immunisations, and others. However, the efficacy of both medication and adjuvant therapy seems to be unsatisfactory, probably because of retained secretions in the airway. Hence, surgery, including FESS and lobectomy, is often indicated after the failure of medication. FESS is reserved for rhinosinusitis and lobectomy for localised lung damage with recurrent haemoptysis or respiratory exacerbations.

FESS may help to improve both upper and lower airway tract infection. It is effective in treating chronic rhinosinusitis that is unresponsive to initial medication, with minimum interruption to normal nasal structure and mucosa. With the development of endoscopic techniques, endoscopic sinus surgery for chronic rhinosinusitis is often indicated for patients with KS (1), even for juveniles (11). In the 2012 European Position Paper on Rhinosinusitis and Nasal Polyps, clinical data showed that FESS had no impact on facial development in children evaluated up to 10 years postoperatively. The use of endoscopic sinus surgery is safe and effective, and is the initial favoured surgical option to reduce disease burden in children with cystic fibrosis-related CRS (12). Similar improvement may be achieved by FESS as the initial surgical option for KS patients with CRS since there are similar clinical characteristics between KS and cystic fibrosis, such as retained secretions, impaired mucociliary clearance, and susceptibility to chronic recurrent airway infections. This surgery aims to establish the drainage of sinuses and improve access for regular cleaning. After surgery, the ethmoid sinus becomes a part of the nasal cavity, and the natural ostium of the frontal sinus is found immediately below the frontal sinus. Gravity drainage of sinus secretions occurs when the head is in an erect position. The sinus cavity of the sphenoid and maxillary sinuses can be easily cleared through the enlarged natural ostia. Additionally, drainage of the maxillary sinus may also occur when the patient is in a lateral position during sleep. Drainage of the sphenoid sinus may occur in the lateral position too, because hypoplasia results in a shallow-bottom shape. FESS is effective not only in treating CRS but also in reducing the incidence of lower-airway infection in KS (13). This is possibly because infected nasal secretions dripping into the lower airway are reduced or stop when rhinosinusitis is controlled. The result of endoscopic sinus surgery in the current case was consistent with the results in previous reports (11). Postoperative debridement of the sinonasal cavity is a part of FESS and plays 
an important role in the management of airway infection in $\mathrm{KS}$. Because the process of postoperative recovery is relatively slow due to impaired mucociliary clearance, the frequency at which debridement is required seems to be higher in KS patients than in patients with common rhinosinusitis after FESS. Therefore, lifelong follow-up is required.

In summary, KS is a rare disease that is often misdiagnosed or diagnosed at a late stage. Herein, we reported a case in which FESS was effective for relieving both CRS and lung infection in the long term. This implies that, although it is a congenital impairment, mucociliary function in the airway could be improved to some extent after effective control of infection and inflammation. Lifelong follow-up is required for extensive recovery of mucociliary function.

Ethics Committee Approval: The study was approved by the local Ethical Committee on Human Experimentation of West China Hospital, Sichuan University.

Informed Consent: Written informed consent was obtained from the patient who participated in the study.

Peer-review: Externally peer-reviewed.

Author contributions: Concept-S.X.L., J.Z., X.H.T.; Design - J.Z., X.H.T. ; Supervision - S.X.L.; Resource - S.X.L., J.Z.; Materials - S.X.L., J.Z.; Data Collection\&/or Processing - X.H.T., J.Z.; Analysis\&/or Interpretation S.X.L., J.Z., X.H.T; Literature Search - J.Z., X.H.T.; Writing - X.H.T., J.Z.; Critical Reviews - S.X.L.

Conflict of Interest: No conflict of interest was declared by the authors.

Financial Disclosure: No financial disclosure was declared by the authors.

\section{References}

1. Cowan MJ, Gladwin MT, Shelhamer JH. Disorders of ciliary motility. Am J Med Sci 2001;321:3-10. [CrossRef]
2. Noone PG, Leigh MW, Sannuti A, Minnix SL, Carson JL, Hazucha $M$, et al. Primary ciliary dyskinesia:diagnostic and phenotypic features. Am J Respir Crit Care Med 2004;169:459-67. [CrossRef]

3. Mittal V, Shah A. Situs inversus totalis:The association of Kartagener's syndrome with diffuse bronchiolitis and azoospermia. Arch Bronconeumol 2012;48:179-82. [CrossRef]

4. Pifferi M, Bush A, Caramella D, Di Cicco M, Zangani M, Chinellato I, et al. Agenesis of paranasal sinuses and nasal nitric oxide in primary ciliary dyskinesia. Eur Respir J 2011;37:566-71. [CrossRef]

5. Scuderi AJ, Harnsberger HR, Boyer RS. Pneumatization of the paranasal sinuses:normal features of importance to the accurate interpretation of CT scans and MR images. AJR Am J Roentgenol 1993;160:1101-4. [CrossRef]

6. Sato I, Sunohara M, Mikami A, Watanabe K, Sato T. Immunocytochemical study of the maxilla and maxillary sinus during human fetal development. Okajimas Folia Anat Jpn 1998;75:205-16.

7. Ellington JK, Elhofy A, Bost KL, Hudson MC. Involvement of mitogenactivated protein kinase pathways in Staphylococcus aureus invasion of normal osteoblasts. Infect Immun 2001;69:5235-42. [CrossRef]

8. Armengot Carceller M, Carda Batalla C, Escribano A, Samper GJ. Study of mucociliary transport and nasal ciliary ultrastructure in patients with Kartagener's syndrome. Arch Bronconeumol 2005;41:11-5. [CrossRef]

9. Campbell R. Managing upper respiratory tract complications of primary ciliary dyskinesia in children. Curr Opin Allergy Clin Immunol 2012;12:32-8. [CrossRef]

10. Boon $M$, Jorissen $M$, Proesmans $M$, De Boeck K. Primary ciliary dyskinesia, an orphan disease. Eur J Pediatr 2013;172:151-62. [CrossRef]

11. Parsons DS, Greene BA. A treatment for primary ciliary dyskinesia:efficacy of functional endoscopic sinus surgery. Laryngoscope 1993;103:1269-72. [CrossRef]

12. Fokkens WJ, Lund VJ, Mullol J, Bachert C, Alobid I, Baroody F, et al. The European Position Paper on Rhinosinusitis and Nasal Polyps 2012. Rhinology Suppl 2012;23:1-299.

13. Wei YX, Xing FH, Miao XT, Liu XC, Zhang X, Lin J. Clinical features of primary ciliary dyskinesia. Zhonghua $\mathrm{Er} \mathrm{Bi}$ Yan Hou Tou Jing Wai Ke Za Zhi 2007;42:195-8. 